\title{
Real-time elastography as a noninvasive assessment of liver fibrosis in chronic hepatitis C Egyptian patients: a prospective study
}

\author{
Lamiaa Mobaraka, Mohammed M. Nabeel ${ }^{b}$, Ehsan Hassan ${ }^{a}$, Dalia Omran ${ }^{b}$, Zeinab Zakaria ${ }^{b}$
}

National Hepatology and Tropical Medicine Research Institute; Cairo University, Cairo, Egypt

\section{Abstract}

\section{Introduction}

Hepatitis $\mathrm{C}$ virus (HCV) is a global disease with serious effects [1]. The highest prevalence of HCV infection (14.7\%) is reported in Egypt [2], mostly by genotype 4 (90\%) [3]. Liver fibrosis is part of the structural and functional alterations in HCV-related chronic liver diseases (CLD). Moreover, in

${ }^{a}$ National Hepatology and Tropical Medicine Research Institute (Lamiaa Mobarak, Ehsan Hassan); ${ }^{\mathrm{b} E n d e m i c ~ M e d i c i n e ~ a n d ~}$ Hepatogastroenterology, Faculty of Medicine, Cairo University (Mohammed M. Nabeel, Dalia Omran, Zeinab Zakaria), Cairo, Egypt

\section{Conflict of Interest: None}

Correspondence to: Zeinab Zakaria, Endemic Medicine \& Hepatogastroenterology Department, Kasr Al Ainy Hospital, Faculty of Medicine, Cairo University, Egypt, Tel.: +201016168831, e-mail: zenab.zakaria@yahoo.com

Received 16 December 2016; accepted 28 March 2016; published online 19 April 2016

DOI: http://dx.doi.org/10.20524/aog.2016.0033 chronic hepatitis $\mathrm{C}(\mathrm{CHC})$, prognosis and management is influenced mainly by the extent of fibrosis [4].

Although liver biopsy remains the gold standard for hepatic fibrosis assessment, it is painful and invasive, with rare but potentially life-threatening complications in addition to some limitations of this technique including interobserver variation and sampling variability which may lead to understaging of cirrhosis [5-7]. Furthermore, liver biopsy cannot be used for mass screening in a country with a very high prevalence of HCV like Egypt.

Limitations of the liver biopsy have motivated research for noninvasive methods for measuring hepatic fibrosis that are less invasive and of equal accuracy [8]. Transient elastography (TE) has emerged as the noninvasive method of reference. It is the most widely used and validated technique that measures liver stiffness based on using elastic shear waves emitted from the vibrator attached to the ultrasound transducer probe. Pulse-echo ultrasound acquisitions follow the shear waves, and the velocity of such waves, directly related to tissue stiffness, is measured. The harder the tissue, the faster the shear wave propagates $[8,9]$. However, it cannot be applied in obese and 
patients with ascites [10]. Failure to obtain any measurement had been reported in $3.1 \%$ of cases and unreliable results (not meeting the manufacturer's recommendations) were reported in $15.8 \%$ [11]. Failure to obtain any measurement and unreliable results rates were $2.7 \%$ and $11.6 \%$ respectively as reported by another study [12].

Real-time elastography (RTE) is technically different from FibroScan. It captures 2-dimensional (2D) strain images induced by internal heartbeats, and the strain images show progressively increasing patchiness with increasing severity of hepatic fibrosis $[13,14]$. Therefore, it can be used in obese patients and those with ascites [15].

In the current study, we aimed to evaluate the value of RTE for the assessment of liver fibrosis in Egyptian patients with $\mathrm{HCV}$-related CLD. RTE results were compared with fibrosis stage obtained by assessing liver biopsy by METAVIR scoring system, TE and aspartate aminotransferase (AST) to platelet ratio index (APRI).

\section{Patients and methods}

This prospective study was conducted in $50 \mathrm{CHC}$ patients, recruited from the outpatient clinics of the National Hepatology and Tropical Medicine Research Institute, Egypt. All patients were positive for $\mathrm{HCV}$ antibodies and $\mathrm{HCV}$ RNA by polymerase chain reaction (PCR). All patients with hepatitis $\mathrm{B}$ virus (HBV) co-infection, decompensated liver disease, hepatocellular carcinoma, history of previous antiviral therapy, body mass index (BMI) $>30$ and presence of absolute contraindication for liver biopsy were excluded from this study. An informed written consent was obtained from all patients according to the 1975 Helsinki Declaration.

All patients were subjected to detailed history, thorough clinical examination, and basic laboratory tests including: complete blood count, AST, alanine aminotransferase, alkaline phosphatase, serum albumin, total bilirubin, INR, a-fetoprotein, hepatitis seromarkers for HCV (anti-HCV) and $\mathrm{HBV}$ (HBsAg, anti HBc, and anti-HBs) using ELISA technique. HCV RNA was tested by quantitative PCR. The APRI index was calculated as follows: AST (/upper limit of normal range) $\times 100$ /platelet count $\left(10^{9} / \mathrm{L}\right)[16]$.

All patients were then subjected to abdominal ultrasonography and liver stiffness measurments using TE (FibroScan, Echosens, France) and RTE (Hitachi, Hi vision Avius, Linear probe (EUP - L 52).

\section{TE}

TE (FibroScan, Echosens, Paris, France) was used following the technical background and examination procedure as described previously [17-19]. Interquartile range was lower than $30 \%$. Results were expressed in kilopascals $(\mathrm{kPa})$. The median value of 10 successful measurements was considered as the representative of the liver stiffness of the median value. All examinations were performed by a single experienced operator.
RTE

RTE (Hitachi, Hi vision Avius, Linear probe EUP - L 52) was used. The tissue elasticity was calculated by the strain and stress of the examined tissue. In first step, the amount of displacement of the reflected ultrasound echoes before and under compression were measured (stress field). In the second step, a strain field was reconstructed from the measured displacements (strain image). High elasticity areas (i.e. soft tissue) showed as places of high strain and low elasticity areas (i.e. hard tissue) showed as places of low strain. Ten valid measurements were performed in each subject and recorded as color-coded images.

Ultrasound-guided liver biopsies were performed within a week of liver stiffness measurements. All samples were examined by a single pathologist, blind to the results of the elastography-based techniques. The grade of activity was evaluated using a modified hepatic activity index: mild (0-6), moderate (7-12) and severe (13-18). Fibrosis was staged according to the METAVIR scoring system from F0 to F4 [20]. Based on the results obtained from histopathological assessment of their liver biopsies, patients were divided into two groups: the significant fibrosis group $(\mathrm{F} \geq 2)(\mathrm{n}=26)$, and the nonsignificant fibrosis group $(\mathrm{F}<2)(\mathrm{n}=24)$.

\section{Statistical analysis}

Continuous data were presented as mean \pm standard deviation while categorical data were presented as number (percent). A P value less than 0.05 was considered statistically significant. All statistical calculations were done using computer programs SPSS (Statistical Package for the Social Science) for Microsoft Windows. The diagnostic performance of TE and RTE were assessed by comparison with liver histology and by measuring the area under the receiveroperating characteristics (AUROC). Diagnostic accuracy was also evaluated by comparing the sensitivity, specificity, positive and negative predictive values (PPV and NPV respectively).

\section{Results}

The present study was conducted on $50 \mathrm{HCV}$ patients. Their age ranged from 27-65 years. The mean age was 44.2 \pm 12 . Demographic features, laboratory data and histopathological features of the included patients are shown in Table 1.

The analysis of the accuracy of RTE, FibroScan and APRI in predicting liver fibrosis in $50 \mathrm{HCV}$ patients is shown in Table 2. At cut-off value $7.5 \mathrm{KPa}$, FibroScan could diagnose significant fibrosis $(\mathrm{F} \geq 2)$ with sensitivity $88 \%$, specificity $100 \%$, PPV 100\%, NPV 89.3\%, and AUROC 0.94 (Table 2, Fig. 1). RTE could diagnose significant fibrosis $(\mathrm{F} \geq 2)$ at cut-off value 2.49 with sensitivity $100 \%$, specificity $66 \%$, PPV $74.6 \%$, NPV $100 \%$, and AUROC 0.8 (Table 2, Fig. 2). APRI, at cut off value 0.65 , could predict significant fibrosis $(\mathrm{F} \geq 2)$ with sensitivity $54 \%$, 
Table 1 Demographic features, laboratory data and histopathological features of the included patients

\begin{tabular}{lc}
\hline Characteristic & $\begin{array}{c}\text { HCV patients } \\
\text { Number }=50\end{array}$ \\
\hline Mean age \pm SD & $44.2 \pm 12.3$ \\
\hline Gender $(\%)$ & \\
\hline Male & $30(60)$ \\
\hline Female & $20(40)$ \\
\hline BMI $\left(\mathrm{Kg} / \mathrm{m}^{2}\right)$ & $25.7 \pm 4.7$ \\
\hline ALT $(\mathrm{U} / \mathrm{mL})$ & $53.8 \pm 24.9$ \\
\hline AST $(\mathrm{U} / \mathrm{mL})$ & $50.8 \pm 21.6$ \\
\hline Albumin $(\mathrm{g} / \mathrm{dL})$ & $4.1 \pm 0.3$ \\
T. Bil (mg/dL) & $0.7 \pm 0.2$ \\
\hline INR & $1.09 \pm 0.1$ \\
\hline AFP (ng/dL) & $6.6 \pm 7.1$ \\
\hline Platelet count (cell/mL) & $209 \times 10^{5} \pm 64.8 \times 10^{5}$ \\
\hline Viral load (IU/mL) & \\
\hline Median (range) & $1.08 \times 10^{5}\left(64-47 \times 10^{6}\right)$ \\
\hline METAVIR (\%) & \\
\hline F0 & $2(4)$ \\
\hline F1 & $22(44)$ \\
\hline F2 & $13(26)$ \\
\hline F2-3 & $4(8)$ \\
\hline & $9(18)$ \\
\hline F4 & \\
\hline
\end{tabular}

Table 2 Diagnostic accuracy of FibroScan, aspartate aminotransferase to platelet ratio index (APRI), real-time elastography (RTE) compared to histopathology in $50 \mathrm{HCV}$ patients in prediction of significant fibrosis $(\geq \mathrm{F} 2)$

\begin{tabular}{lccc} 
& APRI & FibroScan score & RTE \\
\hline Cut-off value & 0.65 & 7.5 & 2.49 \\
SEN (\%) & 54 & 88 & 100 \\
SPE (\%) & 80 & 100 & 66 \\
PPV (\%) & 72.9 & 100 & 74.6 \\
NPV (\%) & 63.5 & 89.3 & 100 \\
LR (+) & 2.7 & - & 2.9 \\
LR (-) & 0.57 & 0.12 & - \\
ACC (\%) & 27.4 & 94 & 83 \\
AUC & 0.69 & 0.94 & 0.8 \\
\hline
\end{tabular}

ACC, accuracy; $L R(+)$, positive likelihood ratio; $L R(-)$, negative likelihood ratio; $N P V$, negative predictive value; $P P V$, positive predictive value; PREV, prevalence of the evaluated fibrosis staging; SEN, sensitivity; $S P E$, specificity, AUC, area under the curve

specificity 80\%, PPV 72.9\%, NPV 63.5\%, and AUROC 0.69 (Table 2, Fig. 3). There was a significant positive correlation between the FibroScan score and RTE score $(\mathrm{r}=0.6, \mathrm{P}=0.001)$ (Fig. 4).

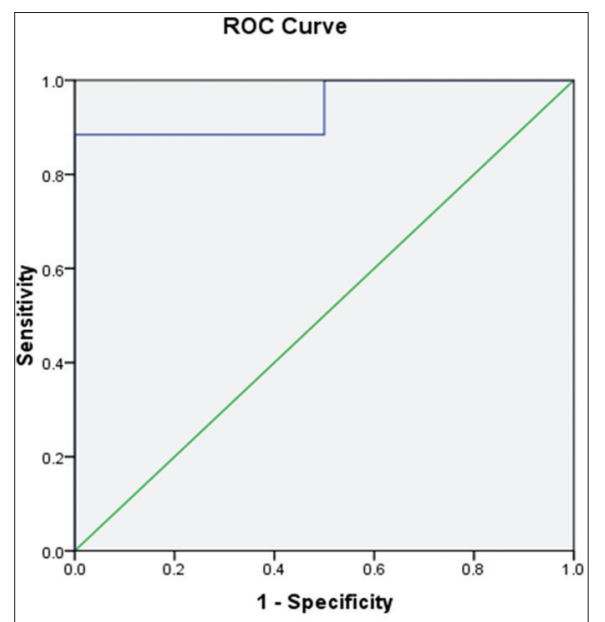

Figure 1 ROC curve for FibroScan

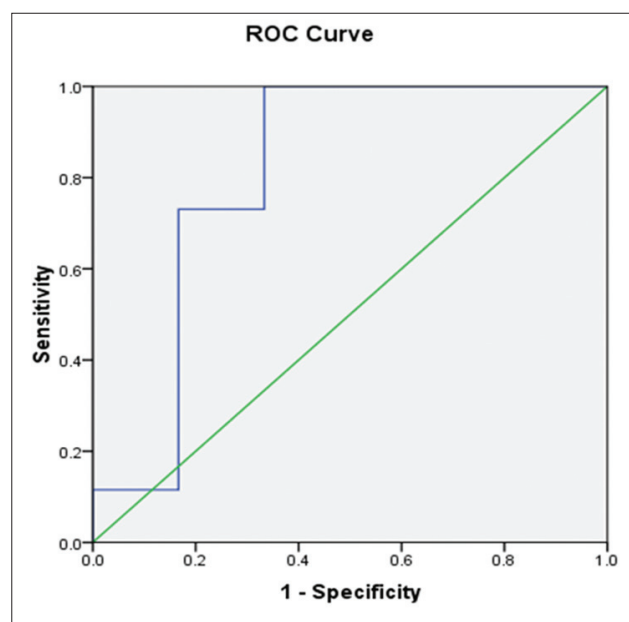

Figure 2 ROC curve for real-time elastography

\section{Discussion}

Ultrasonography-based noninvasive approaches are increasingly considered to assess parenchymal stiffness and progression of CLD [21]. FibroScan measures the propagation speed of shear waves [22-24]. RTE captures 2D strain images induced by internal heart beats, and the strain images show progressively increasing patchiness with increasing severity of fibrosis $[13,14]$. Therefore, it is possible to perform in obese patients or with ascites. In the current study, the diagnostic accuracy of RTE and TE in assessing significant liver fibrosis was compared against histopathology in $\mathrm{CHC}$ patients with $\mathrm{BMI} \leq 30$.

In $\mathrm{CHC}$ patients, prognosis and management strongly depend on the degree of liver fibrosis [4]. Treatment should be initiated promptly in those with severe fibrosis (F3-F4) and should be strongly considered in CHC patients with significant fibrosis $(\mathrm{F}>2)$. Our study showed that TE was able to diagnose the presence of significant fibrosis at a cut off value of $7.5 \mathrm{kPa}$ with a sensitivity of $88 \%$, specificity of $100 \%$, PPV $100 \%$, and NPV $89.3 \%$. The overall accuracy was found to be $94 \%$ with no 


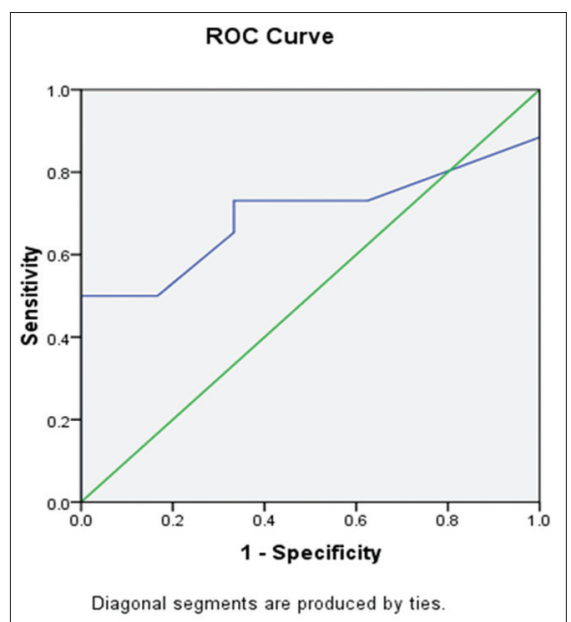

Figure 3 ROC curve for aspartate aminotransferase to platelet ratio index

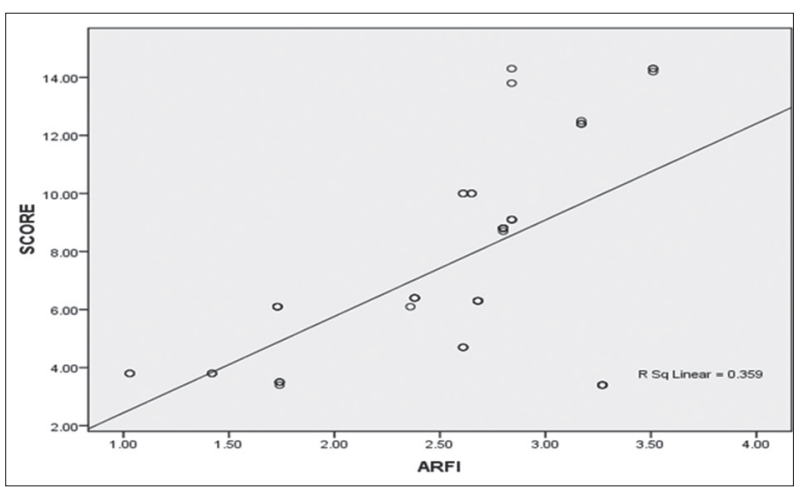

Figure 4 Correlation between real-time elastography and FibroScan score

failure of TE was recorded. This high diagnostic performance of TE was probably explained by exclusion of patients with BMI $>30$ from this study. Likewise, previous studies showed that the AUROCs ranged from 0.79 to 0.83 for the prediction of significant hepatic fibrosis and were over 0.95 for the identification of cirrhosis $[25,26]$.

Other studies reported that TE showed a good diagnostic performance in predicting significant fibrosis, and disease progression to advanced fibrosis and cirrhosis when compared to other noninvasive tests [25,27-30]. Friedrich-Rust et al [29] assessed the overall performance of TE for the diagnosis of liver fibrosis; the AUROCs were 0.84 for significant fibrosis $(\mathrm{F} \geq 2), 0.89$ for severe fibrosis $(\mathrm{F} \geq 3)$, and 0.94 for the diagnosis of cirrhosis $(\mathrm{F}=4)$.

RTE had been reported to be useful for assessment of hepatic fibrosis in patients with $\mathrm{CHC}$ while is not in patients with nonalcoholic fatty liver disease [15]. In the work of Tatsumi et al (2008), RTE correlated well with liver stiffness measured by FibroScan [31]. In our study, RTE was able to diagnose significant fibrosis $(\geq \mathrm{F} 2)$ with a sensitivity of $100 \%$, specificity of $66 \%$, and an overall accuracy of $83 \%$. Although inferior to TE in determining significant hepatic fibrosis, RTE still showed a highly significant positive correlation with TE $(\mathrm{P}=0.001)$. Thus, RTE may be useful for assessing hepatic fibrosis in patients for whom the application of FibroScan may be limited.

FibroScan had some limitations in special patients [32]. Furthermore, examination with FibroScan often requires the use of ultrasonography to find the good window because there is no B-mode and around $20 \%$ of the cases, reliable measurements cannot be obtained by TE using the standard M-probe [11]. On the contrary, RTE displays in real time the relative strain of the tissue by measuring its displacement and it can easily find the most appropriate region and capture the value. Better results may be achieved by a combination of FibroScan (a technology based on shear wave propagation) and RTE (a technology based on tissue distortion).

In the current study, APRI at cutoff value 0.65 could predict significant fibrosis $(\mathrm{F} \geq 2)$ with sensitivity $54 \%$, specificity $80 \%$ and AUROC 0.69; at cut-off 0.5 the sensitivity was $65.4 \%$ and specificity was $66.7 \%$, and at cutoff 1.5 the sensitivity was $30.8 \%$ and specificity of $100 \%$. The diagnostic accuracy of APRI in the current study was lower than previous studies comparing APRI with FibroScan or FibroTest [16,25,33].

Ferraioli et al (2012) suggested that real-time strain elastography can be used in the same way as TE is being used for the assessment of severe fibrosis and cirrhosis, with the benefit of improved assessment of significant fibrosis as no significant difference was observed between AUROCs of TE and real-time SWE for severe fibrosis (0.96 and 0.98, respectively) [34].

In conclusion, although FibroScan is superior in determining significant hepatic fibrosis, our data suggest that

\section{Summary Box}

\section{What is already known:}

- Noninvasive methods for liver fibrosis assessment as ultrasound-based approaches have emerged to replace liver biopsy

- FibroScan is the most widely used and proved technique that measures liver stiffness based on the propagation speed of shear waves

- Real-time elastography (RTE) is technically different (a technology based on tissue distortion)

\section{What the new findings are:}

- The overall diagnostic accuracy of FibroScan was found to be $94 \%$, while in RTE it was $83 \%$

- Although RTE was less than FibroScan in determining significant liver fibrosis, our data suggest that RTE may be a useful for liver fibrosis assessment in chronic hepatitis $\mathrm{C}$ patients especially in cases with technical limitations for FibroScan

- There was a significant positive correlation between the FibroScan score and RTE score $(r=0.6$, $\mathrm{P}=0.001$ ) 
RTE may be a useful and promising noninvasive method for liver fibrosis assessment in CHC patients, especially in cases with technical limitations for FibroScan.

\section{ACKNOWLEDGMENT}

All authors would like to thank Dr Mohamed Hassany for his cooperation and paying much effort to regulate the work

\section{References}

1. WHO | Hepatitis C. World Health Organization; 2012; (Fact sheet no.164).

2. Mohamoud YA, Mumtaz GR, Suzanne R. and Miller D. The epidemiology of hepatitis $C$ virus in Egypt: a systematic review and data synthesis. BMC Infect Dis 2013;13:288.

3. Nguyen MH, Keeffe EB. Prevalence and treatment of hepatitis C virus genotypes 4, 5, and 6. Clin Gastroenterol Hepatol 2005;3(Suppl 2):S97-S101.

4. European Association for the Study of the Liver. EASL Clinical Practice Guidelines: management of hepatitis C virus infection. J Hepatol 2011;55:245-264.

5. Friedman SL, Bansal MB. Reversal of hepatic fibrosis-fact or fantasy? Hepatology 2006;43:82-88.

6. Bravo AA, Sheth SG, Chopra S. Liver biopsy. $N$ Engl J Med 2001;344:495-500.

7. Colloredo G, Guido M, Sonzogni A. Impact of liver biopsy size on histological evaluation of chronic viral hepatitis: the smaller the sample, the milder the disease. J Hepatol 2003;39:239-244.

8. Foucher J, Chanteloup E, Vergniol J. (FibroScan): Diagnosis of cirrhosis by transient elastography (FibroScan): a prospective study. Gut 2006;55:403-408.

9. Takeda T, Yasuda T, Nakayama Y. Usefulness of noninvasive transient elastography for assessment of liver fibrosis stage in chronic hepatitis C. World J Gastroenterol 2006;12:7768-7773.

10. European Association for the Study of the Liver. J Hepatology 2015;63:237-264.

11. Castera L, Foucher J, Bernard PH, et al. Pitfalls of liver stiffness measurement: a 5-year prospective study of 13,369 examinations. Hepatology 2010;51:828-835.

12. Wong GL, Wong VW, Chim AM, et al. Factors associated with unreliable liver stiffness measurement and its failure with transientelastography in the Chinese population. J Gastroenterol Hepatol 2011;26:300-305.

13. Friedrich-Rust M, Ong M, Herrmann E, et al. Real-time elastography for noninvasive assessment of liver fibrosis in chronic viral hepatitis. AJR 2007;188:758-764.

14. Morikawa H, Fukuda K, Kobayashi S, et al. Real-time tissue elastography as a tool for the noninvasive assessment of liver stiffness in patients with chronic hepatitis C. Gastroenterology 2011;46:350-358.

15. Tomeno W, Yoneda M, Imajo K, et al. Evaluation of the liver fibrosis index calculated by using real-time tissue elastography for the non-invasive assessment of liver fibrosis in chronic liver diseases. Hepatol Res 2013;43:735-742.

16. Wai CT, Greenson JK, Fontana RJ, et al. A simple noninvasive index can predict both significant fibrosis and cirrhosis in patients with chronic hepatitis C. Hepatology 2003;38:518-526.

17. Ziol M, Handra-Luca A, Kettaneh A, et al. Noninvasive assessment of liver fibrosis by measurement of stiffness in patients with chronic hepatitis C. Hepatology 2005;41:48-54.

18. Castera L, Forns X, Alberti A. Non-invasive evaluation of liver fibrosis using transient elastography. J Hepatol 2008;48:835-847.

19. Kettaneh A, Marcellin P, Douvin C, et al. Features associated with success rate and performance of fibroscan measurements for the diagnosis of cirrhosis in HCV patients: a prospective study of 935 patients. J Hepatol 2007;46:628-634.

20. Bedossa P, Poynard T. The METAVIR cooperative study group. An algorithm for the grading of activity in chronic hepatitis C. Hepatology 1996;24:289-293.

21. Paparo F, Corradi F, Cevasco L, et al. Real-time elastography in the assessment of liver fibrosis: a review of qualitative and semiquantitative methods for elastogram analysis. Ultrasound Med Biol 2014;40:1923-1933.

22. Ferraioli G, Tinelli C, Dal Bello B, Zicchetti M, Filice G, Filice C. Accuracy of real-time shear wave elastography for assessing liver fibrosis in chronic hepatitis C: a pilot study. Hepatology 2012;56:2125-2133.

23. Friedrich-Rust M, Wunder K, Kriener S, et al. Liver fibrosis in viral hepatitis: noninvasive assessment with acoustic radiation force impulse imaging versus transient elastography. Radiology 2009;252:595-604.

24. Sandrin L, Fourquet B, Hasquenoph J, et al. Transient elastography: a new noninvasive method for assessment of hepatic fibrosis. Ultrasound Med Biol 2003;29:1705-1713.

25. Castera L, Vergniol J, Foucher J, et al. Prospective comparison of transient elastography, Fibrotest, APRI, and liver biopsy for the assessment of fibrosis in chronic hepatitis C. Gastroenterology 2005;128:343-350.

26. Ziol M, Handra-Luca A, Kettaneh A, et al. Noninvasive assessment of liver fibrosis by measurement of stiffness in patients with chronic hepatitis C. Hepatology 2005;41:48-54.

27. Shaheen AA, Myers RP. Diagnostic accuracy of the aspartate aminotransferase-to-platelet ratio index for the prediction of hepatitis C-related fibrosis: a systematic review. Hepatology 2007;46:912-921.

28. Poynard T, Morra R, Halfon P, et al. Meta-analyses of FibroTest diagnostic value in chronic liver disease. BMC Gastroenterol 2007;7:40.

29. Friedrich-Rust M, Ong MF, Martens S, et al. Performance of transient elastography for the staging of liver fibrosis: a metaanalysis. Gastroenterology 2008;134:960-974.

30. Castèra L, Le Bail B, Roudot-Thoraval F, et al. Early detection in routine clinical practice of cirrhosis and oesophageal varices in chronic hepatitis C: comparison of transient elastography (FibroScan) with standard laboratory tests and non-invasive scores. J Hepatol 2009;50:59-68.

31. Tatsumi R, Kudo M, Ueshima K, et al. Noninvasive evaluation of liver fibrosis Using serum fibrotic markers, transient elastography (FibroScan) and real-time tissue elastography. Intervirology 2008;51(Suppl 1):27-33.

32. Fraquelli M, Rigamonti C, Casazza G, et al. Reproducibility of transient elastography in the evaluation of liver fibrosis in patients with chronic liver disease. Gut 2007;56:968-973.

33. Le Calvez S, Thabut D, Messous D, et al. The predictive value of Fibrotest vs. APRI for the diagnosis of fibrosis in chronic hepatitis C. Hepatology 2004;39:862-863.

34. Ferraioli G, Tinelli C, Malfitano A, Dal Bello B, Filice G, Filice C. Liver Fibrosis Study Group, Above E, Barbarini G, Brunetti E, et al. Performance of real-time strain elastography, transient elastography, and aspartate to- platelet ratio index in the assessment of fibrosis in chronic hepatitis C. AJR Am J Roentgenol 2012;199:19-25. 